\title{
A mayor IMC mayor grado de insatisfacción de la imagen corporal.
}

\author{
Artículo Original
}

Miguel Casillas-Estrella, Nancy Montaño-Castrejón, Vanessa Reyes-Velázquez, Montserrat BacardíGascón, Arturo Jiménez-Cruz.

\section{RESUMEN.}

Antecedentes. Las presiones sociales provocan que las personas fuera de la "norma" establecida por la sociedad, presenten diversos grados de insatisfacción de la imagen corporal (IIC). Entre las consecuencias de la IIC se señalan el aislamiento, la baja autoestima, la ansiedad y la depresión.

Objetivo. El objetivo del presente estudio es valorar el grado de IIC de personas que acuden a un centro de atención primaria universitaria en Tijuana.

Metodología. Ciento cincuenta y ocho pacientes de una clínica de primer nivel participaron en el estudio. Se les solicitó a los participantes que indicaran sobre el pictograma de Stunkard, la figura con la que identifican su imagen corporal personal y la imagen corporal personal deseable. A todos los participantes se les tomó el peso, la estatura y la circunferencia de cintura. Para valorar si había una asociación entre índice de masa corporal (IMC) y el grado de IIC se usó la correlación de Spearman. Para observar las diferencias de grado de IIC con riesgo de obesidad abdominal, sobrepeso, obesidad, género y antecedentes de obesidad infantil se realizaron pruebas de t para muestras independientes.

Resultados. Se observó una correlación (Spearman) positiva entre el grado de insatisfacción y el IMC (O.544, P < 0.01). Quienes presentaron un peso adecuado tuvieron una IIC promedio de 0.9, quienes presentaron sobrepeso de1.7 y quienes presentaron obesidad de 2.6. Las mujeres presentaron un mayor grado de IIC (2.0) que los hombres (1.5) $(\mathrm{p}<0.01)$, las personas con riesgo de exceso de grasa abdominal $(\mathrm{P}<0.0001)$, con sobrepeso y con obesidad ( $\mathrm{P}<0.001)$, y las personas que referían sobrepeso antes de los 15 años.

Recomendaciones. Son necesarias medidas integrales de prevención y control del exceso de grasa corporal en diferentes contingencias, niveles, estructuras, instituciones, y sectores. Asimismo, se deben controlar diversos factores de riesgo en todos los períodos de la vida.

(Rev Biomed 2006; 17:243-249)

Palabras clave: obesidad, insatisfacción de la

Solicitud de sobretiros: Arturo Jiménez-Cruz. Programa de Postgrado en Nutrición. Facultad de Medicina, Universidad Autónoma de Baja California. Calzada Tecnológico 14418, Tijuana, B.C. 14390, México. Tel. 664 6821233, Correo electrónico: ajimenez@uabc.mx

Recibido el 12/Septiembre/2006. Aceptado para publicación el 6/Noviembre/2006.

Este artículo está disponible en http://www.uady.mx/sitios/biomedic/revbiomed/pdf/rb061742.pdf

Vol. 17/No. 4/Octubre-Diciembre, 2006 


\section{Casillas-Estrella, N Montaño-Castrejón, V Reyes-Velazquez, M Bacardí-Gascón y col.}

imagen corporal, estigmatización, autoestima.

\section{SUMMARY.}

Higher BMI means greater body image dissatisfaction.

Background. Social pressure causes people outside the "norm" established by society to develop different degrees of body image dissatisfaction (BID). Consequently, different psychosocial alterations such as isolation, low self-esteem, anxiety, and even depression could result.

Objective. The objective of this study was to determine the degree of BID in people who attend a primary care university center.

Method. One hundred and fifty eight patients seeking medical assistance agreed to participate in this study. They were asked to select a figure from the modified Stunkard pictogram, which they most believed to represent their personal body image and their desired body image. Weight, length, and waist circumference were obtained from all the participants. A Spearman correlation was utilized to determine if there was an association between body mass index (BMI) and the degree of BID; ttests for independent samples were used to assess the differences of BID and abdominal obesity, overweight, obesity, gender, and history of obesity before age 15 .

Results: A positive correlation between degree of BID and BMI (O.544, P < 0.01) was shown. Normal BMI had an average BID score of 0.9, overweight 1.7, and obese 2.6. Women $(\mathrm{P}<0.01)$ as well as people with abdominal adiposity $(\mathrm{P}<$ $0.0001)$, or who were overweight or obese ( $\mathrm{P}<$ 0.001 ), as well as those with childhood history of fat excess had higher degrees of BID.

Recommendations. It is necessary to establish comprehensive obesity prevention programs including different levels of contingencies, structures, institutions, sectors and risk factors, in all stages of life.

(Rev Biomed 2006; 17:243-249)
Keywords: obesity, body image dissatisfaction, estigmatization, self-esteem.

\section{INTRODUCCIÓN.}

La imagen corporal personal corresponde a la imagen mental de uno mismo hacia su cuerpo. Factores socioculturales influyen sobre la percepción de la imagén corporal. Se ha sugerido que a mayor nivel socioeconómico y de conocimientos se presenta una mayor incidencia de la imagen corporal personal distorsionada (ICPD) $(1,2)$.

El estigma es la adjudicación a una persona o grupo de una característica que representa una identidad devaluada en un contexto social específico. Es decir, los estigmas son representaciones de una sociedad que tiene percepciones negativas sobre grupos particulares (1), de tal manera que los individuos de una sociedad pueden considerar que las personas con exceso de grasa corporal son responsables de su gordura. Este tipo de estigmatización tiene su origen en la edad media, cuando consideraban como causa principal de la obesidad a la pereza, holgazanería y la glotonería (3).

Las personas más expuestas al estigma del peso pueden ser vulnerables a efectos psicológicos como la depresión y sociales como el aislamiento (1). Algunos autores consideran que la estigmatización hacía las personas con exceso de grasa podría ser una de las actitudes más aceptables en la sociedad (4).

Las consecuencias de los prejuicios hacia la persona estigmatizada, como el aislamiento, pueden contribuir a la exacerbación del sobrepeso, como resultado del aumento del consumo de alimentos de alto contenido energético para compensar la ansiedad, o la disminución de la actividad física para evitar la exposición social a las burlas o señalamientos $(1,5)$. Incluso algunos estudios reportan que estos individuos presentan una mayor incidencia de depresión, nerviosismo, tabaquismo y alcoholismo desde

\section{Revista Biomédica}




\section{Obesidad e insatisfacción de la imagen corporal}

edades tempranas (6).

Aunque la evidencia empírica sugiere que el peso corporal esta determinado por una compleja interacción de factores biológicos y ambientales, existe información que sugiere que un gran porcentaje de la población piensa que la falta de voluntad y control individual es la causa principal del exceso de grasa corpporal (1). También se ha considerado el resultado de una mala higiene personal, de una actitud hostil o de actitudes deshonestas (7). Por otro lado, no solamente individuos obesos muestran insatisfaccion de la imagen corporal (IIC). Diversos estudios sugieren que los individuos con extrema delgadez, con anorexia nerviosa o con bulimia, también presentan cierto grado de IIC y de ICPD. En general, las figuras corporales más aceptadas son aquellas que corresponden a las figuras 3, 4 y 5 del Pictograma de Stunkard adaptado por Rand. (8).

En México algunos estudios han demostrado que los niños y adolescentes mexicanos prefieren la amistad de un niño con peso normal y seleccionan al niño con sobrepeso en último lugar, entre niños con seis diferentes características físicas $(9,10)$. También se ha descrito una actitud negativa de los médicos hacía las personas con sobrepeso (11). Sin embargo, no hemos encontrado estudios que valoren el grado de satisfacción de la imagen corporal en población mexicana.

El objetivo del presente estudio es valorar el grado de IIC de personas que acuden a un centro universitario de atención primaria.

\section{Metodología.}

Lugar

Baja California es un estado al noroeste de la República Mexicana colindante con el estado de California, en los Estados Unidos. Según el censo del 2000 contaba con una población aproximada de 2,487,367 habitantes, siendo Tijuana la ciudad más grande del estado y una de las cinco ciudades más grandes de México (12).

Población

El presente estudio se realizó en el Centro
Universitario Médico Asistencial y de Investigación (CUMAI) de la Universidad Autónoma de Baja California en Tijuana, de enero a mayo del 2006. Proceso

Después de proporcionarles información sobre el propósito del estudio, a todos los pacientes mayores de 18 años que acudieron a consulta de 8: 00 am a 12:00pm, se les solicitó su autorización para participar en el estudio. Tres estudiantes de último año de la carrera de medicina (pasantes) participaron como entrevistadores.

Durante este período se atendieron un total de 371 pacientes que acudieron a asistencia médica de primer nivel, de los cuales sólo 158 (43\%) aceptaron participar en el estudio.

\section{Colección de Datos}

Se les solicitó a los participantes que indicaran sobre el pictograma modificado de Stunkard (8), la figura con la que identificaban su imagen corporal personal (ICP) y la imagen corporal personal deseable (ICPD).

\section{Reproducibilidad}

Se presentó el pictograma de Stunkard modificado (8) en dos ocasiones en un período de tres semanas. Se realizó el procedimiento de test-retest y el grado de concordancia para la evaluación del peso deseable fue superior a 0.8 (p $<0.05$ ).

Medición Antropométrica

Los encuestadores realizaron la medicion del peso y talla con un estadiómetro portátil marca Detecto, NIST 641345, hecho en Webb City MO, EEUU. La calibración de la báscula la realizó la compania Básculas Internaciones de Mexico a 0.1 $\mathrm{kg}$.

Se obtuvo el índice de masa corporal (IMC) con la fórmula: peso (kg) / estatura ${ }^{2}\left(\mathrm{~m}^{2}\right)$.

La circunferencia de cintura se midió en el punto medio entre la cresta ilíaca y el borde inferior de última costilla.

Criterios de sobrepeso, obesidad y obesidad abdominal

Los criterios para valorar el sobrepeso fueron $\geq 25$ a $30 \mathrm{~kg} / \mathrm{m}^{2}$ y de obesidad $\geq 30 \mathrm{~kg} / \mathrm{m}^{2}$, para

Vol. 17/No. 4/Octubre-Diciembre, 2006 


\section{Casillas-Estrella, N Montaño-Castrejón, V Reyes-Velazquez, M Bacardí-Gascón y col.}

evaluar alto riesgo de exceso de grasa abdominal, fueron $\geq 88 \mathrm{~cm}$ para las mujeres $\mathrm{y} \geq 102$ para los hombres (13).

Clasificación de Grado de Insatisfacción de la Imagen Corporal (IIC)

Para evaluar el grado de IIC se calculó de la manera siguiente: IIC=ICP- ICPD. Se consideró satisfecho cuando ICP-ICPD $=0$; ligeramente insatisfecho cuando ICP-ICPD $=1$; moderadamente insatisfecho cuando ICP-ICPD= 2; y severamente insatisfecho cuando ICP-ICPD $\geq 3$. No se incluyeron los que se consideraban insatisfechos por bajo peso (3 personas con peso adecuado).

Análisis Estadístico

Los datos fueron analizados utilizando el programa SPSS para Windows 11.5. Para valorar si había una relación entre IMC y el grado de IIC se ejecutó la correlación de Spearman. Para observar las diferencias de grado de IIC con riesgo de obesidad abdominal, sobrepeso, obesidad, género y antecedentes de obesidad infantil se realizaron pruebas de t para muestras independientes. Se realizó análisis de varianza y la prueba para comparaciones múltiples de Bonferroni para analizar las diferencias entre insatisfacción y categorías de IMC.

\section{RESULTADOS.}

Del total de participantes, 113 (72\%) correpondían al sexo femenino y 39 (28\%) al masculino. El promedio de edad de los participantes fue de 41.5 (18 a 78) años. La prevalencia de sobrepeso fue de $41 \%$ y la de obesidad 39\%. La prevalencia de riesgo de exceso de grasa abdominal fue de $62 \%$ y dos porciento (63\% las mujeres y 58\% los hombres). El 22\% de los participantes refierieron haber presentado sobrepeso u obesidad desde antes de los 15 años.

La distribución del grado de IIC se presenta en el cuadro 1. En general, el $84 \%$ de la población tenía algún grado de IIC. Entre las personas con peso adecuado, el $12 \%$, presentaban IIC, 92\%, quienes tenían sobrepeso, y 98\% quienes presentaron obesidad el 98\% (cuadro 2). De acuerdo al pictograma de Stunkard, en el $62 \%$ de las personas con algún grado de IIC, la diferencia fue de dos o más figuras y fue mayor en las personas con sobrepeso y obesidad (figura 1).

Se observó una correlación (Spearman) positiva entre el grado de insatisfacción y el IMC (O.544, P < 0.01). Además se observaron diferencias estadísticamente siginificativas entre el grado de IIC y los diferentes criterios de exceso de grasa corporal: quienes presentaron un peso adecuado tuvieron una IIC promedio de 0.9, quienes presentaron sobrepeso de 1.7 y quienes presentaron obesidad de 2.6. Se observaron diferencias estadísticamente significativas de IIC entre cada categoría de IMC. Las mujeres presentaron un mayor grado de IIC (2.0) que los hombres (1.5) $(\mathrm{p}<0.01)$, también las personas con riesgo de exceso de grasa abdominal $(\mathrm{P}<$ 0.0001 ), con sobrepeso y con obesidad ( $\mathrm{P}<$ 0.001 ), y las personas que referían sobrepeso antes de los 15 años $(\mathrm{P}<0.005)$.

Cuadro 1

Grado de Insatisfacción de la Imagen Corporal

\begin{tabular}{|l|lc|}
\hline & $\mathrm{N}$ & $(\%)$ \\
\hline Satisfecho & 25 & $(16)$ \\
\hline Insatisfacción ligera (+1) & 23 & $(15)$ \\
\hline Insatisfacción moderada (+2) & 65 & $(42)$ \\
\hline Insatisfacción severa $(\geq 3)$ & 40 & $(26)$ \\
\hline Total & 100 & \\
\hline
\end{tabular}

\section{Revista Biomédica}


Obesidad e insatisfacción de la imagen corporal

\section{Cuadro 2}

Indice de Masa Corporal e Insatisfacción de la Imagen Corporal.

\begin{tabular}{|c|c|c|c|c|c|c|}
\hline IMC (Peso/Talla $\left.{ }^{2}\right)$ & $\begin{array}{l}\text { Hombres } \\
\mathrm{N} \quad(\%)\end{array}$ & $\begin{array}{c}\text { GIIC } \\
\%\end{array}$ & $\begin{array}{l}\text { Mujeres } \\
\text { N (\%) }\end{array}$ & $\begin{array}{c}\text { GIIC } \\
\%\end{array}$ & $\begin{array}{l}\text { Total } \\
\text { N (\%) }\end{array}$ & $\begin{array}{c}\text { GIIC } \\
\%\end{array}$ \\
\hline $18.5-24.99$ & (18) & 5 & $24(21)$ & 15 & 32 (20) & 12 \\
\hline $25-29.99$ & $16 \quad(36)$ & 88 & 49 (43) & 94 & $65(41)$ & 92 \\
\hline$\geq 30$ & $20 \quad(46)$ & 100 & $41(36)$ & 98 & 61 (39) & 98 \\
\hline$\geq 25$ & $36 \quad(82)$ & 94 & 90 (79) & 96 & $126(81)$ & 95 \\
\hline
\end{tabular}

GIIC: Grado de Insatisfacción de la Imagen Corporal

\section{DISCUSIÓN.}

En este estudio se observó que $84 \%$ de los participantes que presentaron algún grado de IIC por exceso de peso. En promedio la imagen corporal percibida por los participantes en este estudio podría corresponder con el alto porcentaje de sobrepeso y obesidad observados en esta población. Estos resultados son similares a los observados por Mirza y cols (2005), quienes encontraron que las personas con sobrepeso y obesidad presentaron un mayor grado de IIC (14). En otro estudio donde se evaluó la percepcion del peso en relacion a "los kilos de más o de menos", la mayoría de los participantes subestimaban su peso con relación a los criterios internacionales de peso ideal $\left(18.5-25 \mathrm{~kg} / \mathrm{m}^{2}\right)$ (15).

En este estudio se presentó un grado de IIC moderado o severo en más del 65\%. de los participantes. Más alto en mujeres, en personas con obesidad abdominal, con sobrepeso u obesidad y en aquellas personas que iniciaron el sobrepeso antes de los 15 años. Resultados que son similares a otros estudios (16) en población angloamericana. Algunos estudios han descrito

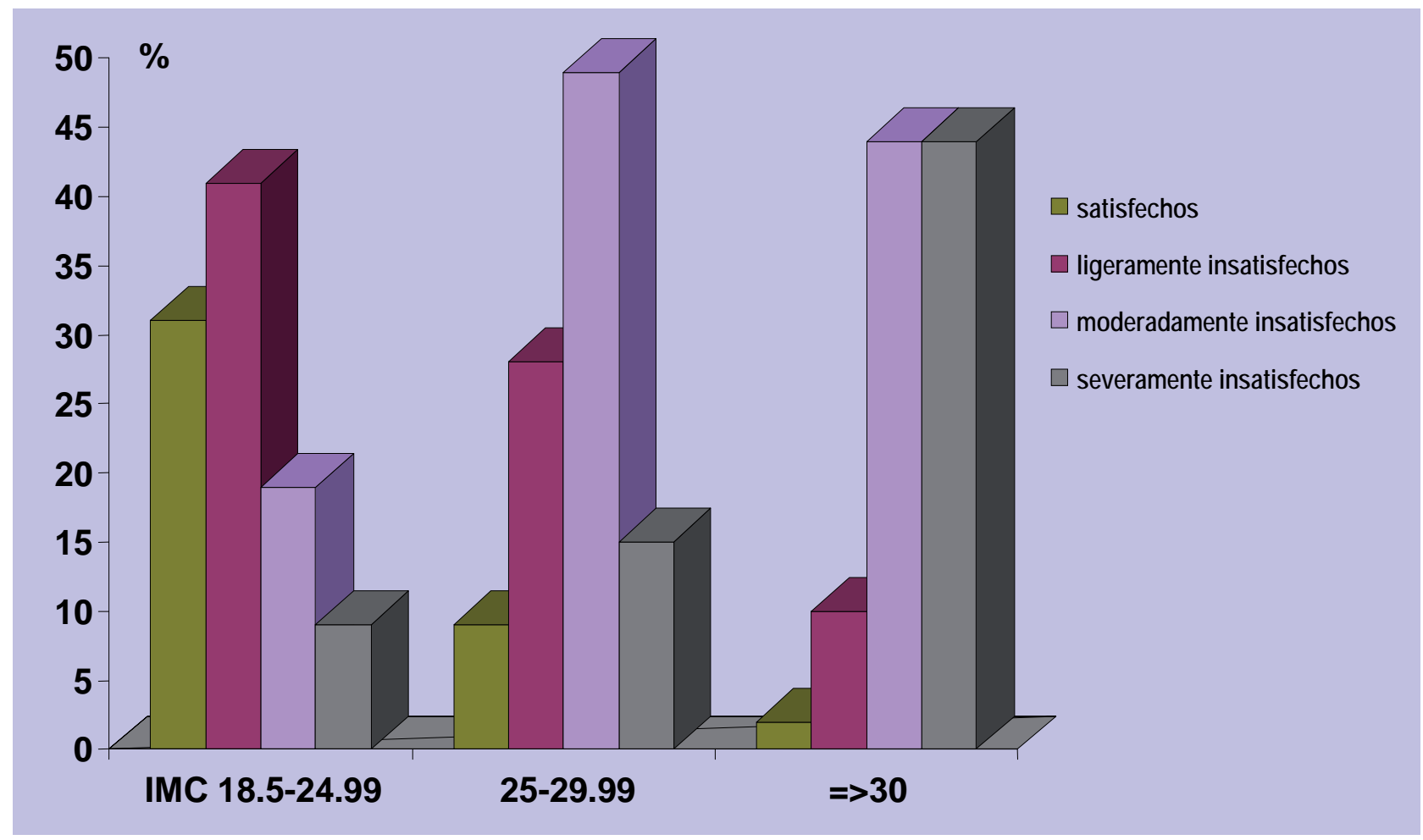

Figura 1.- Índice de Masa Corporal $\left(\mathrm{kg} / \mathrm{m}^{2}\right)$ y Grados de Insatisfacción de la Imagen Corporal 


\section{Casillas-Estrella, $N$ Montaño-Castrejón, V Reyes-Velazquez, M Bacardí-Gascón y col.}

que personas afroamericanas, especialmente las mujeres, tiene menor insatisfacción de la imagen corporal equivalente al sobrepeso, cuando se les compara con otros grupos étnicos $(17,18)$. En los EEUU se ha observado que los individuos hispanos presentan un menor grado de IIC en relación con los de origen caucásico, pero mayor que los afroamericanos (19). Sin embargo, en este estudio podemos observar que más del $90 \%$ de quienes presentaron sobrepeso y obesidad tuvieron un alto grado de IIC.

En otros estudios se han observado diferencias en la satisfacción de la imagen corporal de acuerdo al género. En la ciudad de México, se ha descrito en niños y jovenes, que las mujeres presentan mayor grado de IIC por sobrepeso u obesidad, y los hombres mayor preocupacion por la corpulencia (20). Sin embargo, el promedio de edad de los hombres de este grupo fue de 41 años, por lo que parece que, en el caso de los mexicanos, a mayor edad menor preocupación por la corpulencia. El grado de IIC por exceso fue mayor en las mujeres.

A pesar de que hay estudios que describen menor grado de IIC en la población hispana, lo observado en este estudio sugiere que la influencia de los medios de comunicación y de la fuerte relación económica, social y cultural entre la población norteamericana y la residente de la frontera norte de México, tiene un gran impacto. Lo que podría resultar en problemas psicosociales por baja autoestima, ansiedad y depresion de la mayoría de las personas con exceso de grasa corporal $(1,14)$. Queda por determinar si la población en la frontera mexicana es más susceptible a la influencia de los medios y a la moda norteamericana que la población hispana residente en los EEUU o que la mexicana de otras partes del país. El hecho de que la IIC sea mayor en personas que iniciaron el sobrepeso antes de los 15 años sugiere, como se ha observado en otros estudios (16), que el tiempo de exposición a burlas de sus pares puede tener un efecto sobre la satisfacción y la autoestima (23). Es por esto que las medidas de prevención del sobrepeso deberían iniciar desde la edad escolar.

Las limitantes de este estudio fueron que aunque el pictograma utilizado presentó una confiabilidad mediante la prueba test-retest superior a 0.8 , no se realizó con ese instrumento la validación sobre el grado de IIC en poblacion mexicana. La población de estudio corresponde a personas que acuden a un centro de atención primaria (principalmente dirigido a personas con bajos ingresos), lo que no representa a la población en general.

En conclusión, en esta poblacion existe un alta prevalencia de sobrepeso u obesidad (81\%), las mujeres presentaron mayor riesgo de exceso de grasa abdominal y mayor grado de IIC que los hombres, a mayor sobrepeso más grado de IIC en población, y a menor edad de inició de la obesidad mayor grado de IIC. Estos resultados son alarmantes en una sociedad que tiene una de las prevalencias más altas de obesidad. Medidas integrales a diferentes niveles (individual, familiar, comunitario, municipal, estatal y nacional), estructuras (ambiente físico, estado socioeconómico), instituciones (gobiernos locales, estatales, federales), sectores (educativo, salud, social) y sobre diversos factores (dieta, actividad física, estrés, hambre) deben aplicarse con urgencia en todas las etapas de la vida.

\section{REFERENCIAS.}

1.- Puhl R, Brownell K. Psychosocial origins of obesity stigma: toward changing a powerfull and pervasive bias. International Association for the Study of Obesity, Obes Rev 2003; 4:213-27.

2.- Guinn B, Semper T, Jorgensen L, Skaggs S. Body image perception in female Mexican-American Adolescents. J Sch Health 1997; 67:112-5.

3.- Stunkard A. Stigmatization of obesity in medieval times. Asia and Europe. Int J Obes 1998; 22:1141-4.

4.- Puhl R, Brownell K. Bias, Discrimination and obesity. Obes Res 2001; 9:788-805.

\section{Revista Biomédica}


Obesidad e insatisfacción de la imagen corporal

5.- Wardle J, Waller J, Fox E. Age of onset and body dissatisfaction in obesity. Addict Behav 2002; 27:561-73.

6.- Strauss R. Childhood obesity and self esteem. Pediatrics. 2000; 105; 1:e15.

7.- Teachman B, Brownell K. Implicit anti-fat bias among health professionals: is anyone immune? Int J Obes 2001; 25:1525-31.

8.- Rand C, Wright B. Continuity and change in the evaluation of ideal and acceptable body sizes across a wide age span. Int J Eat Disord 2000; 28:90-100.

9.- Jimenez Cruz A, Leon J, Bacardí-Gascón M. Gender and ethnic differences of Mexican children's attitudes toward a drawing of an obese peer. Asia Pac J Clin Nutr 2004; 13 (Suppl):S142.

10.- Castellon A, Bacardí M, Jiménez Cruz A. Perception of healthy and obese Mexican teenagers towards their obese peer. Obes Res 2004; 12 (Suppl): A72-P274.

11.- Jimenez Cruz A, Garcia JL, Castellon, A. Bacardí M. Attitudes toward obese adults and children among Mexican teachers, parents, and health personnel. Obes Res 2005;13 (Suppl): A155-601P.

12.- Instituto Nacional de Estadistica. Articulo en linea. Disponible en http:www.inegi.gov.mx. Accesado en Julio 2006.

13.- Expert Panel on the Identification, Evaluation, and Treatment of Overweight Adults. Clinical Guidelines on the Identification, Evaluation and Treatment of Overweight and Obesity in Adults: Executive Summary. Am J Clin Nut 1998;68:899-917.

14.- Mirza N, Davis D ,Yanovsi J.Body dissatisfaction, selfesteem, and overweight among inner-city Hispanic children and adolescents. J Adolesc Health 2005; 36:267e16-20.

15.- Gila A, Castro J, Toro J, et al. Subjective body image dimensions in normal female population: Evolution through adoloscence and early adulthood. Int J Psychol and Psychol Ther 2004; 4:1-10.

16.- Wardle J, Waller J. Fox E. Age of onset and body dissatisfaction in obesity. Addictive behaviors.2002;27: 561-73.

17.- Bhuiyan A, Gustat J, Srinivasan S, Berenson G. Differences in body shape representations among young adults from a biracial (black-white), semirural community. Am J Epidemiol 2003; 158:792-7.

18.- Welch C, Gross S, Bronner Y, Dewberry-Moore N, Page DM. Discrepeancies in body imagen perception among fourth-grade public school children from urban, suburban, and rural Maryland. J Am Diet Assoc 2004; 104:1080-5.

19.- Guinn B, Semper T, Jorgensen L, et al. Body image perception in female Mexican-American adolescents. J Sch Health 1997; 67:112-5.

20.- Vazquez R, López X, Alvarez G, Mancilla J, Oliva A. Insatisfaccion corporal e influencia de los modelos esteticos en ninos y jovenes varones mexicanos.Enseñanza e investigacion en psicologia.2006;11;1:185-97.

21.- Leonhard M, Barry J. Body image and obesity:effects of gender and weight on perceptual measures of body image.Addict Behav 1998; 23:31-4.

22.- Sánchez-Johnsen L, Fitzgibbon M, Martinovich Z, Stolley MR, Dyer AR, Van Horn L. Ethnic differences in correlates of obesity between Latin-American and Black Women. Obes Res 2004; 12:652-60.

23.- Jackson TD, Grilo CM, Masheb RM. Teasing history, onset of obesity, current eating disorder psychopathology, body dissatisfaction, and psychological functioning in binge eating disorer. Obes Res 2000; 8:451-8. 